\title{
Best Papers of the 2016 ACM Multimedia Systems (MMSys) Conference and Workshop on Network and Operating System Support for Digital Audio and Video (NOSSDAV) 2016
}

The 7th ACM International Conference on Multimedia System (MMSys'16) was successfully held in Klagenfurt am Wörthersee, Austria, from May 10-13, 2016, (http:// mmsys2016.itec.aau.at) with the co-located workshops NOSSDAV, MoVid, and MMVE.

ACM MMSys 2016 introduced a new format referred to as overview talks, which have been held on the first conference day starting in the afternoon and concluding in the evening with a get-together event at the conference venue. The following overview talks have been given at MMSys: "Using Games to Solve Challenging Multimedia Problems" by Oge Marques, ACM Distinguished Speaker, FAU, USA; "More Juice Less Bits: MediaMelon Content-Aware Streaming" by Ali C. Begen, MediaMelon Inc., USA, Ozyegin University, Turkey, IEEE ComSoc Distinguished Lecturer, "MPEG-DASH Spatial Relationship Description" by Omar Aziz Niamut, TNO, The Netherlands, "Mulsemedia: Novelty or Reinvention?" by Gheorghita Ghinea, Brunel University, UK; and "Smart Camera Systems" by Bernhard Rinner, Alpen-Adria-Universität Klagenfurt, Austria.

ACM MMSys typically comes with keynotes from experts and leaders in industry and academy. The first keynote was about "Ten Thousand Channels to Ten Million Viewers: Technologies for Scaling Video Delivery over IP" by Neill A. Kipp, Comcast VIPER, USA, addressing issues with video delivery at scale, whereas the second keynote entitled "Advances and Trends in Augmented Reality Systems" by Dieter Schmalstieg, Graz University of Technology, Austria, was related to one of the special session. The third keynote was about "5G Enabling the Tactile Internet" by Frank Fitzek, Technische Universität Dresden, Germany, providing insights about next-generation mobile networks.

In general, ACM MMSys 2016 attracted 71 full paper submissions, from which 20 got finally accepted in the program, which has been carefully selected from our experienced members of the technical program committee. In addition to the full paper submissions, MMSys 2016 hosted two special sessions, one on augmented reality and another on media synchronization. A demo session provided researchers, engineers, and scientist to present the opportunity to showcase their research prototypes, systems, and applications to MMSys attendees. An important aspect of MMSys is the dataset track, which enables reproducible research thanks to the availability of common datasets across different application areas. In particular, the dataset track is an opportunity for researchers and practitioners to make their work available and citable.

This special section is dedicated to extended versions of the best paper and best student paper from MMSys and-for the first time-the papers awarded with the Excellence in DASH Award sponsored by the DASH-IF. Finally, this section also includes the best paper from NOSSDAV.

The first paper-the MMSys Best Paper-by Stefano D'Aronco et al. is entitled "Distributed Rate Allocation in Switch-Based Multiparty Videoconferencing Systems" and proposes a distributed algorithm to allocate the bitrates in a quality-of-service (QoS) aware manner that can reallocate bitrates due to changes in the context conditions.

2017 Copyright is held by the owner/author(s).

1551-6857/2017/06-ART40

DOI: http://dx.doi.org/10.1145/3084539

ACM Trans. Multimedia Comput. Commun. Appl., Vol. 13, No. 3s, Article 40, Publication date: June 2017. 
The algorithm has been evaluated through simulation and benefits are highlighted in terms of network usage and average utility compared to a baseline method.

The second paper-the MMSys Best Student Paper-by Luca De Cicco et al. discusses "Design and Performance Evaluation of Network-assisted Control Strategies for HTTP Adaptive Streaming." In this paper, the authors implement a video control plane, which enforces video quality fairness among concurrent video flows generated by heterogeneous client devices. The proposed approach is evaluated with respect to several quality-of-experience metrics such as video quality fairness, video quality, and switching frequency as well as the impact of the client-side adaptation algorithm.

The Excellence in DASH Award was selected by members of the DASH-IF and the first price has been distributed equally among the following three papers. In "On Optimizing Adaptive Algorithms based on Rebuffering Probability," Andrzej Beben et al. present a new adaptation and buffer management algorithm that selects video representations based on the predicted probability of video freezing. Its efficiency has been evaluated through simulation and trial experiments. Jan Willem Kleinrouweler et al. propose "An SDN Architecture for Privacy-Friendly Network-Assisted DASH," which adopts the SDN paradigm for DASH-based media delivery to increase video bitrate and at the same time reduces quality switches. Cong Wang et al. discuss "Design and Analysis of QoE-Aware Quality Adaptation for DASH: A Spectrum-Based Approach," which provides a significant reduction of number and magnitude of quality switches that has been evaluated within a cross-Atlantic internet scenario.

Finally, Cong Zhang et al. present-the NOSSDAV Best Paper-entitled "CloudAssisted Crowdsourced Livecast," which provides a measurement and analysis of hybrid cloud-assisted crowdsources live streaming.

In closing, we would like to thank the authors for their efforts in preparing the manuscripts for this special section. We would like to thank the reviewers of MMSys and NOSSDAV, members of the award committees, and the actual reviewers of this special section for their valuable comments throughout the review process and important contributions toward producing this special section.

\author{
Christian Timmerer \\ Alpen-Adria-Universität Klagenfurt/Bitmovin, Austria \\ Ali C. Begen \\ Networked Media/Ozyegin University, Turkey
}

